\title{
THE SOCIETY OF BRITISH NEUROLOGICAL SURGEONS:
}

\section{3rd MEETING}

The 63rd meeting of the Society was held on May 12 and 13, 1961, at the Rigshospitalet, Copenhagen, Denmark. The President, Mr. D. W. C. Northfield, was in the chair.

\section{The Incidence of Cerebral Ischaemia in Spontaneous Subarachnoid Haemorrhage}

R. CAMpbell Connolly (London) said that as infarction of the brain had been reported in a high proportion of cases coming to necropsy after spontaneous subarachnoid haemorrhage, and as marked narrowing of the main brain arteries was commonly seen on angiography during the first three weeks following such haemorrhage, he had made an attempt to discover how frequently severe clinical signs were due to ischaemia.

In a consecutive and personal series of 136 patients, $73(54 \%)$ had at some time during the acute phase of their illness and before operation developed well-marked evidence of a focal lesion of the brain as shown by dysphasia, visual field defects, hemiparesis or akinetic mutism. Thirty of these patients proved to have sizeable intracerebral haematomas but in the remaining 43 no evidence of a haematoma was discovered and the signs were considered to be due to ischaemia.

It was assumed that the arterial narrowing, almost certainly due to spasm, occurred acutely at the time of a haemorrhage. It might be partly responsible for stopping the haemorrhage, but it had less desirable effects and, by suddenly reducing blood flow to the brain, might be the cause of loss of consciousness in some cases when bleeding was confined to the subarachnoid space. Afterwards it remained a potent factor in increasing morbidity and mortality. This was demonstrated by a striking difference in the mortality rates in the three clinical groups of patients. In the whole series of 136 patients there were 24 deaths $(18 \%)$; of the 43 patients with focal ischaemic lesions of the brain, 18 died $(43 \%)$; of the 30 with haematomas, five died $(17 \%)$; while of the 63 remaining cases only one died $(1.6 \%)$ from uraemia due to polycystic disease of the kidneys. The overall mortality rate after operation designed to prevent further haemorrhage was $8.3 \%$ in 60 cases.

It was concluded that ischaemia of the brain played a vital role during the acute phase following spontaneous subarachnoid haemorrhage and might constitute a hazard perhaps as great as the risk of recurrent haemorrhage. Efforts should, therefore, be made at every stage before, during, and after operation to combat the complication.

\section{Cerebral Apoplexy: Surgical Treatment of Intracranial Haematomas}

J. RIISHEDE (Copenhagen) said that in Denmark the annual number of apoplectic seizures was estimated at a minimum of 30,000. Deaths from cerebral apoplexy amounted to $100-125$ per 100,000 individuals, i.e., 5-6,000 each year, of whom $25 \%$ were under 65 years of age. In a consecutive series of 300 patients admitted to a neurosurgical department for cerebral apoplexy the frequency of intracerebral haematomas was $25 \%$.

Seventy-four of 98 patients with cerebral apoplexy due to intracerebral haematomas were operated on. Fortyfive were alive six months after operation and 26 of these had normal or slightly reduced working capacity at the follow-up from one to 10 years after operation.

Cerebral Apoplexy: Role of Carotid Occlusion in the Necko

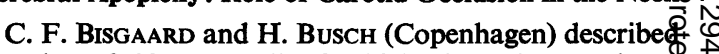
a series of 29 cases all of which showed stenosis o@ occlusion of the carotid arteries under arteriography ${ }_{\mathcal{O}}$ Nineteen were operated on and 10 treated conservatively॰ All patients had anticoagulation therapy. Heparin was given as an intravenous drip in acute cases. Secondarp haemorrhage occurred in two cases in which intermitten heparin was used. A shunt was best established by us $\overrightarrow{0}$ of a stiff, looped, large-diameter polythene tube place in both ends of the arterial incision. This permitted increased working space and a reduction of the clamping time to four or five minutes. The shunt was most important when dealing with a stenosis, but they established it if they obtained a back-flow from a total occlusion.

Grafts were not used but in some cases the distal part of the incision was sutured transversely. Two cases not included in the series were presented, one a case of traumatic thrombus, and one with a kink of the internal carotid artery. Their results were not good but were not more discouraging than those obtained with conservative therapy. However, they still preferred to operate, especially on those patients with stenosis, because they felt it was better to discharge a patient with two functioning carotids.

They concluded that if operation for patients with occlusion were to be performed, then it should be immediate. In less acute cases of stenosis they preferred to start anticoagulation therapy before operation.

\section{Cerebral Embolectomy}

R. MALmRos (Aarhus) reported on a series of four cases operated on for occlusion of the middle cerebral artery since 1956. Three were due to emboli and one to thrombosis. 
In two cases the emboli were removed through an incision in the middle cerebral artery and the arterial wall resutured. There was immediate filling of peripheral branches but no clinical improvement. The patients died six days and six weeks after operation. In the one thrombotic case the patient died on the table.

The fourth case was a woman of 29 with mitral stenosis. Embolism occurred six hours before operation. She had severe neurological signs corresponding to occlusion of the middle cerebral artery with conjugate deviation of the eyes, homonymous hemianopia, severe hemiplegia, hemianaesthesia, and absent stereognostic and position sense. The embolus occupied $10 \mathrm{~mm}$. length of the middle cerebral artery but arteriography showed fairly good peripheral collateral circulation. At operation a temporary clip was placed on the internal carotid artery and the embolus removed through the anterior cerebral artery after this had been divided a few millimetres from the internal carotid artery. There was good back flow and clips were placed on the stumps of the anterior cerebral artery and the clip removed from the internal carotid. Post-operative angiography showed good filling of all the middle cerebral branches. Angiography 18 days after operation showed slight spasm in the internal carotid and the posterior cerebral arteries. She recovered slowly after the operation but two and a half months later there were no abnormal signs and mentally there was only slight euphoria, fatuity, and impairment of memory.

He concluded that it might be difficult to remove an embolus through an incision in the middle cerebral artery but that it could be removed through the anterior cerebral when it was possible to divide this without serious consequences. The results might be good even when several hours had elapsed since the embolism.

\section{Continuous Recording of Ventricular Pressures in Neuro- surgical Practice}

NiLS LUNDBERG (Lund) reported on a method for the continuous recording of ventricular fluid pressure. Its applicability as a routine aid in the treatment of intracranial lesions and intracranial hypertension of other origin was evaluated on the basis of his experience in 130 cases. In 51, recordings were continued for more than a week and in 17 for more than three weeks. The material was studied with respect to infection, tissue reaction, and intracranial haemorrhage. There was no evidence that the ventricular puncture or the indwelling catheter were responsible for any significant complications.

In cases of intracranial hypertension records showed a great variety of spontaneous fluctuations amongst which three main types could be distinguished. (1) Rhythmic oscillations of $\frac{1}{2}$ to 2 per minute and amplitude between 10 and $30 \mathrm{~mm}$. $\mathrm{Hg}$. These were found to be related to periodic breathing of the Cheyne-Stokes type and synchronous variations of the alveolar $\mathrm{CO}_{2}$ content. (2) Waves with frequency of 4 to 8 per minute and amplitude less than $20 \mathrm{~mm}$. $\mathrm{Hg}$ were related to similar fluctuations in systemic blood pressure. (3) Plateau waves occurring at irregular intervals with amplitude of 50 to $100 \mathrm{~mm} . \mathrm{Hg}$ and lasting a few minutes up to hours were also noted. These were associated with headaches, impairment of consciousness, nausea and vomiting, flushing of the face, and motor phenomena such as rigidity and clonic movements of the limbs. These attacks were similar to those described previously as cerebellar or tonic fits, diencephalic fits, etc. It was stressed that these waves might be premonitory signs of hypertensive episodes after pneumography or acute swelling of the brain during operation and might be followed by signs of irreversible brain damage. Intermittent obstruction of the cerebrospinal fluid did not appear to be necessary to produce these waves and they were seen in diffuse tumour infiltrations with large ventricles and free subarachnoid spaces. E.E.G. recording during these waves showed no epileptic activity.

Plateau waves were only seen in association with a raised mean ventricular pressure, disappeared after temporal decompression, and could be induced by raising the ventricular pressure. He considered that they were manifestations of a critical instability of the mechanism controlling the reciprocal relationship between cerebral blood flow and intracranial pressure.

He concluded that continuous graphic recording of ventricular pressure could yield valuable information in the treatment of patients and offered the possibility of recording ventricular pressures for long periods without undue risk. Its practical value was increased by the fact that it included a rational and relatively safe procedure for ventricular drainage and pneumography.

\section{Observations on the Use of a Cytotoxic Agent in the Treatment of Malignant Brain Tumours}

W. Atrinson (London) said that certain pharmaceutical manufacturers had begun trials with cytotoxic agents for the treatment of malignant tumours. The team at the Imperial Chemical Industries' pharmaceutical division had selected an epoxide, 32865, after animal trials, for use in man. The author confirmed their findings as to toxicity in monkeys.

Three patients suffering from cerebral gliomata were given different courses of injections of the compound into the common carotid artery after ligation of the external branch. The first two patients died but the third continued to live a somewhat sheltered life after seven months' treatment.

Details of histology, arteriographic pattern, and haematology were discussed. The outcome of the use of the compound in cases with intracranial sarcoma was mentioned. The dangers and discomfort attendant on this form of treatment and the need for careful selection of patients and regular control of blood cell levels were emphasized.

\section{The Place of Decompression of the Posterior Root in the Treatment of Trigeminal Neuralgia}

P. TAARnhøJ (Copenhagen) discussed the present position of decompression of the posterior root for trigeminal neuralgia which he had introduced 10 years ago. He stated that the procedure could be carried out by the temporal intradural or extradural route or by a posterior fossa approach. It was important to manipulate the posterior root and in his series this had been done by 
running a nerve hook along the root. The importance of this manipulation had first been pointed out by Sheldon.

His series consisted of 155 patients of whom four had been treated bilaterally. The mortality had been $0.5 \%$ and the complications equally low. Patients had been followed up for as long as 10 years with an average of 6.5 years. Three patients had no relief; in one this was due to technical difficulties. Ninety patients $(60 \%)$ had now had no complaints. Fifty-four $(35 \%)$ had had a recurrence of pain and five had uncharacteristic sensations in the face. Almost half of the recurrences took place during the first year following surgery and $80 \%$ in the first three years. Following recurrence 21 patients had section of the posterior root and of these $37 \%$ were painfree and in $23 \%$ pain had recurred. Fourteen patients had a second decompression and half of these were painfree, the other half having had a second recurrence. He felt that following recurrence a second decompression produced better results than root section.

He pointed out that other workers had not had as good results, their recurrence rate in several series varying from none to $75 \%$. He felt that some of the bad results might have been due to not manipulating the nerve at the time of operation.

He then reviewed reports of follow-up studies after various other procedures such as alcohol injection and posterior root section a:d noted that there were very few of these reports available. He concluded that the results of root section or alcohol injection were not as good as many people imagined and that the conservative procedure called decompression or compression should be considered when a patient with trigeminal neuralgia asked for relief.

\section{Osteo-arthritis of the Mandibular Joint and Facial Pain}

P. RASMuSSEn (Aarhus) presented material consisting of 146 patients with facial pain admitted to the State Department of Neurosurgery in Aarhus during a period of four years. Of these patients, 60 had trigeminal neuralgia, 69 atypical neuralgia, and 17 atypical facial pain.

He said that trigeminal neuralgia and osteo-arthritis of the mandibular joint had the following things in common: age distribution, sex distribution, seasonal variation and influence of the temperature, attacks of pain precipitated by functional activity, pain at first movements, and the effects of osteo-arthritic treatment.

By the objective as well as by the radiographic examination the patients with facial pain more frequently presented symptoms of osteo-arthritis than those without facial pain. Most of the patients had arthritic changes in the same side as the facial pain.

All the 146 patients were treated with intra-articular injections with hydrocortisone. In $64 \%$ they had an immediate effect. One half of the recurrences set in during the first three months. Six months after treatment, $25 \%$ of the entire series and $38 \%$ of the group with immediate effect were without pain. One year after, the numbers were $19 \%$ of the entire series and $30 \%$ of the group with immediate effect. The patients with trigeminal neuralgia had the best effect. Patients with positive and negative findings of osteo-arthritis had the same effect.
Hydrocortisone was used as a 'test treatment'. Further으 examinations would be necessary to judge of the $Z$ importance of these results.

Extradural Haematoma at the Vertex

Presented by G. Alexander (Bristol).

\section{Occult Forms of Spinal Dysraphism}

L. P. LASS 1 AN and Michael JAMES (Newcastle) $\stackrel{\bigcirc}{?}$ discussed their experience with certain forms of congenital spinal lesions. They considered that the original. term, myelodysplasia, covered too many conditions and $\Rightarrow$ was no longer specific; most of them could be grouped $\stackrel{\oplus}{\stackrel{\oplus}{+}}$ under the heading of spinal dysraphism which indicatedo their common origin from failure of the embryo to으 develop completely in the median dorsal region. This term, therefore, included all forms of spina bifida and $\overparen{\nabla}$ each case would be found to be a variation in degree of abnormality from the open myelocele at one extreme to $\omega$ the smallest failure of fusion of the spinous process at the $\overrightarrow{0}$ first sacral vertebra at the other.

Spina bifida was common and it had been shown that $\overrightarrow{\vec{\omega}}$ certain progressive foot deformities in children, associated $\Omega$ with this anomaly, were due to congenital lesions of the cord or cauda equina and it had been possible to make? an early diagnosis in these cases. The symptoms and ${ }_{\perp}$ signs conformed to a pattern which allowed the formula $\vec{i}$ tion of a syndrome with characteristic myelographiei findings. The most important finding on myelograpl was the level of the conus medullaris, and where the confof was abnormally low a lesion had always been found. present they held the view that there would not beç surgically treatable congenital malformation of the spinal cord or cauda equina without a laminal defect.

Thirty cases had been submitted to operation. Twent four had presented as an orthopaedic syndrome, 13 wiकh $\vec{\omega}$ external manifestations. Three had presented as an external manifestation and three as a neurological bladder. Laminectomy confirmed the diagnosis and revealed lesions that could be shown to act in the following ways: Two cases had pressure bands, 15 cases had traction $\bar{\partial}$ bands, with associated intrathecal lipomata in five cases. Combined traction and pressure bands were found in one $\mathbb{D}$ case and diastomatomyelia in 10 cases. (This abnormality was present in 12 cases.) No abnormality was found in 음 two. The lesions were found to be amenable to surgical treatment and, although the intention had been to prevent further deterioration, there had been noticeable improvement in two-thirds of the cases.

The Neurological Aspects of Retroperitoneal Malignancy

A. Hulme (Bristol) said that infiltrating tumours in the retroperitoneal tissues might produce a syndrome closely resembling a primary intraspinal lesion such as cauda equina tumour or prolapsed intervertebral disc. An

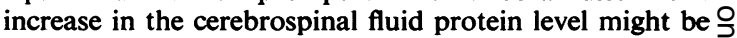
found even when the spinal canal and vertebra were not $D$ directly involved.

Three illustrative case histories were presented. In two, dubiously abnormal myelographic appearances prompted $\mathcal{N}$ a laminectomy, with negative findings.

Although the possibility of retroperitoneal disease was $N$ 
suspected in each instance, this was not clinically detectable when the patients were first seen.

The finding of an abdominal or pelvic mass was delayed in the first case for two and a half months, and in the second and third for nine months. One patient proved to be suffering from an ovarian carcinoma and one from a fibrosarcoma. The third case was diagnosed clinically as a neurofibrosarcoma but this was not confirmed histologically. An interesting feature was the increase in C.S.F. protein level in the absence of a spinal subarachnoid block or direct involvement of the vertebral canal. Suggested explanations were that this was attributable to an aseptic inflammatory response in the involved nerve roots, or perhaps to interference with venous drainage from the epidural space.

\section{The Early Diagnosis of Acoustic Neuromas}

WYLIE MCKISSOCK (London) said the high mortality rate associated with total excision of acoustic neuromas was largely due to late diagnosis. With early diagnosis the mortality rate should be almost zero. During the period 1940-60 he had operated upon 270 acoustic neuromas but in only eight of these patients was the tumour less than $3 \mathrm{~cm}$. in diameter. With these small tumours the operation was one of no greater difficulty than section of the eighth or ninth nerves.

Of the eight patients, five were men and three women and all complained of progressive deafness. Six complained of attacks of vertigo and six of tinnitus. Only two had noted disturbance of sensation in the face and one patient had noticed twitching of one side of the face during a two-week period.

Neurological signs were limited to the cranial nerves, all patients having some degree of deafness, six some disturbance in function of the fifth nerve but only a partial seventh nerve palsy.

Radiographs of the skull showed an enlarged porus in only two cases. In five patients the cerebrospinal fluid was examined, the protein being normal in three and 65 and $110 \mathrm{mg}$. respectively in the other two.

Air studies were performed in every case, found negative in seven but outlining the tumour in the remaining case.

Special otological testing by Dr. C. S. Hallpike or Mr. Terence Cawthorne indicated the presence of the tumour and on this evidence and clinical suspicion operation was undertaken. Once the exposure was made the tumour could be separated from the eighth nerve with a blunt hook and removed.

There was no fatality and recovery was rapid in all cases. In five cases eighth nerve function was retained, three having no increase in the pre-operative degree of deafness. In the remaining three patients hearing was lost although the nerve was macroscopically intact. Several patients showed a transient seventh nerve weakness but all recovered in a few weeks or months.

He concluded that the greatest publicity should be given in medical, neurological, and otological circles to the need for early diagnosis of these lesions by special otological tests: only in this way would the mortality from operation be reduced to a negligible level.
Experience with the Sptiz-Holter Valve in the Treatment of Hydrocephalus

V. Knudsen and P. HedegaARd (Copenhagen) reported 46 cases having ventriculovenous shunts. Seventeen cases were of communicating hydrocephalus, 18 were of obstructive hydrocephalus (10 with aquaduct stenosis, eight with tumours), eight cases had myelomeningocele and three cases miscellaneous diagnoses. Thirty-four cases had functioning shunts. All patients had been followed from one to 24 months post-operatively. An analysis of the failures was given.

\section{Chemopallidotomy and Chemothalamotomy in Parkinson's Disease}

B. BROAgER and T. NORHøLM (Copenhagen) reported on a series of 100 consecutive patients with Parkinsonism treated by chemopallidotomy or thalamotomy. Cooper's technique was followed except that small successive injections were given instead of balloon inflation. All patients were operated on before April, 1960, the followup time being one to four years.

The average age was 56 years, 37 being over 60 . Sixty-three patients had unilateral and 37 bilateral treatment; several patients had more than two lesions. Five patients died within eight weeks of operation and were considered surgical mortality. Some of these went into coma after pneumography and introduction of the cannula into the medial pallidum. Although no injections were given and the cannula withdrawn the following day, coma lasted until death. Necropsy showed no evidence of haemorrhage but severe diffuse degeneration in the basal ganglia. Thirteen patients died after longer periods of time and four could not be traced. The remaining 78 patients were followed up.

The immediate post-operative effect, lasting more than three months, was very good or good in 85 patients but in 10 the operation had a poor effect or no effect. The effect on rigidity was very good or good in 80 patients (84\% of 95 patients who had rigidity). There was little or no effect in 10 patients.

Severe complications included three cases of deep haematoma with lasting hemiplegia (two of these were included in the late deaths). In several patients with bilateral treatment a disturbance of speech was noticed after the second operation, mostly an aphonia.

In the follow-up at one to four years a good or very good effect on tremor was found in $58(60 \%), 10$ had recurrence of tremor, and 13 had died. A very good or good effect on rigidity was seen in 61 patients $(66 \%$ of those with rigidity) and in 12 it showed little or no effect.

An estimate had been made of the overall condition of the patients and it was found at follow-up that 45 cases were benefited, 21 were no better than before operation, and 12 were worse. In addition 18 patients had been improved for substantial periods of time but had later deteriorated from progression of disease (13) or had died of the disease (2) or of some other illness (3). In all, 63 $(66 \%)$ were improved for long periods of time or were still improved at follow-up. Amongst the 32 patients not improved in their overall condition, including the five operative and seven late deaths, 15 were completely or 
severely oligokinetic before operation and four others were prematurely aged and partly demented. Such patients would not be operated on at the present time. The best results would be obtained by operating on goodrisk patients and on one side only, but in spite of this, several bad-risk patients in the series had had good and lasting results from treatment.

\section{The Use of Subdural Air Injections in the Selection of Patients with Subfrontal Fractures for Operation}

ANTONY JefFerson and Norman Lewtas (Sheffield) stated that because meningitis resulting from anterior fossa fractures might be fatal in spite of prompt treatment, it was vital to recognize the presence of dural lacerations adjacent to the nasal cavity or to the frontal, ethmoidal, or sphenoidal sinuses.

The surgeon treating anterior fossa fractures might need to operate before rhinorrhoea had been observed or meningitis had occurred. In a series of 13 patients who had required dural grafts in the frontal region, three had never experienced rhinorrhoea, no intracranial air had been observed, and meningitis had not developed. The authors described and illustrated a method by which air, injected subdurally through a frontal drill hole, might be used to demonstrate adhesions between the floor of the skull and the under surface of the frontal lobe. In the presence of subfrontal fractures, such adhesions corresponded to the site of dural defects.

By means of subdural injections the surgeon might sometimes be able to satisfy himself that there was no dural defect, and hence an unnecessary operation could be avoided. Absence of a dural defect might be demonstrated even though, in the initial phases, air had been detected within the skull, or the radiological features of the fracture made an accompanying dural laceration highly probable. Examples of these two situations were illustrated.

It was emphasized that the present contribution represented a preliminary communication.

\section{Lumbar Myelography with Water-soluble Contrast}

H. H. JACOBSEN (Copenhagen) reported on the use of Abrodil in lumbar myelography. He said that in cases with cervical or thoracic localization he used gas myelography except in patients who were obese, had pronounced scoliosis, or cardiac disease. In these cases Pantopaque was used.

In using Abrodil for lumber myelography a spinal anaesthetic was necessary and blood pressure must be checked during and after operation. During the period 1959-60, 200 such examinations had been carried out. In 29 cases the examination was reported as normal; in three of these a subsequent operation was performed, and in only one was a disc prolapse found. In 142 cases a disc prolapse was diagnosed and 117 were operated on. A prolapse at the level expected was found in 104 cases and prolapses at two levels which had been predicted in five cases. Protrusions were found in three and only exostoses without prolapse in two. In three cases onlye arachnoiditis or scar tissue was found. There were now negative explorations. In 18 of the 142 myelographies? only a shortening of the root sheath was found withour filling defect in the contrast column. Ten of these werę operated on and prolapse found at the expected level. In 19 cases a protrusion was diagnosed and of eight patients $\widehat{乛}$. operated on only three showed a prolapse. In the remain ing 10 cases miscellaneous conditions were diagnosed. One had scar tissue and five sacral root cysts. Two had: cystic malformations (one verified) and one had a tumouf? (verified). One had a large terminal sac which made judgment as to disc prolapse impossible and proved tô have a prolapse at operation.

Complications occurred in 14 cases $(7 \%$ ) but none wereD permanent. Three patients had slight headache; 10 ha क् severe pain in the legs or perineal region, and seven of these recovered at once after Pethidine. One patient haø an epileptic fit 24 hours after myelography.

\section{Local Cooling as an Adjunct to Cerebral Operations}

K. Kristiansen (Oslo) and N. M. DotT (Edinburgh) in presenting a preliminary report on a case in which lccal cerebral cooling was employed, referred briefly tọ the history of hypothermia and its recent use as adjunct to cerebral surgery. They expressed the view twato profound cooling was desirable, and contrasted $\overrightarrow{\mathrm{g}} \mathrm{ts}$ achievement in the brain by the method of profout general cooling involving cardiac by-pass with profoundlocal cerebral cooling involving carotid by-pass only. pioneer work had been done by $K$. Kristiansen gim developing and practising the latter method with exceltents results.

The case reported was one of right Sylvian arterioven malformation in a youth of 16 years. The fistulous ffow was exceptionally large and there were special difficulties of access to the vessels concerned. Dr. Kristiansen ha\& been consulted and he and Professor Semb of Oslo haळ generously agreed to receive the patient, Professor Dotto and a team of four others, in order that the operation would be carried out in Oslo with the benefits of the locaß cerebral cooling technique developed there. This wast successfully accomplished in December, 1960. A slight residual hemiparesis was believed to be due to arteria spasm and temporary local ischaemia in the field of the middle cerebral basal perforating arteries proximal to the lesion and to the site of temporary occlusion of the middle cerebral artery. The lesion was probably incurred by reason of persistent spasm of these small branches aftep the cold perfusion had ceased and the brain had been re-warmed. Possible means to overcome this risk by the local application of papaverine to the arterial walls was discussed. It was suggested that this adjunct to cerebrab surgery had many advantages and that it could and should be perfected by further laboratory work and by cautious clinical application. 